\title{
Alteration of the Aggregation Stability of Quantum Dots by Irradiation
}

\author{
Aleksey Tsipotan $^{1, \mathrm{a})}$, Aleksandr Aleksandrovsky ${ }^{1,2 \mathrm{~b})}$ and Vitaliy Slabko ${ }^{1, \mathrm{c})}$ \\ ${ }^{1}$ Siberian Federal University, Krasnoyarsk 660041, Russia. \\ ${ }^{2}$ Russian Academy of Sciences, Kirensky Institute of Physics Krasnoyarsk 660036, Russia. \\ a) Corresponding author: cipotana@mail.ru \\ b) aleksandrovsky@kirensky.ru \\ c)vslabko49@mail.ru
}

\begin{abstract}
The influence of weak irradiation on the aggregation stability of quantum dots is considered. It was shown that under combined UV and visible light irradiation, a gradual increase in spontaneous aggregation takes place, testifying decrease in stabilizing potential barrier height. Thereby, the laser-induced controllable self-assembly of CdTe QDs is recommended to be performed over a time period between 80 and $100 \mathrm{~min}$ after the beginning of low intensity irradiation under conditions equivalent to those applied in this study.
\end{abstract}

\section{INTRODUCTION}

Production of nanostructures consisting of semiconductor nanoparticles (NPs), or QDs, is of interest for number of applications [1-6]. Development of new methods of QDs' manipulation and aggregation of QDs into nanostructures with pre-defined geometry is also of considerable interest from the fundamental point of view. Under laser irradiation at the wavelengths falling into excitonic absorption, polarization of semiconductor QDs at optical frequency will be induced. Electrodynamical interaction between polarized NPs is rather universal and allows formation of wide variety of nanostructures both of homo- and heterogeneous content. Certain universality of available configurations can be achieved using electrodynamical approach of creating the nanostructures with the pre-defined geometry, as considered in [7, 8]. This approach is based on laser frequency and polarization dependent attraction of nanoparticles that leads to their controllable self-assembly. Smart choice of potential barrier in the vicinity of QD's surface is important for this method since that barrier must not only combat the spontaneous aggregation, but it is extremely important for the geometry of obtained nanostructures.

One of possible ways to control the barrier height is the illumination of colloidal QDs' solutions by weak optical radiation preliminarily to laser-induced self-assembly. The influence of weak optical irradiation on the aggregation of colloid solutions is well known. E. g., the spectral dependence of the photostimulated aggregation rate of colloidal silver nanoparticles was investigated in [9]. The mechanisms leading to the decrease of the height of the barrier preventing the spontaneous aggregation for the case of silver nanoparticles were discussed in [10], and, as the rule, they are connected with the electron photoemission from metallic NP. Similar studies concerning the control of aggregation stability of semiconductor QDs under the irradiation in various spectral ranges are absent.

In the present paper we study the possibility to alter the aggregation stability of QDs' solutions by weak UV and visible irradiation. 


\section{MATERIALS AND METHODS}

Solution of CdTe QDs (average diameter $2.9 \mathrm{~nm}$, purchased from PlasmaChem Gmbh, Berlin, Germany) in water was used as the object. QDs were stabilized against spontaneous aggregation by thioglicole acid. Molar concentration of QDs in the solution was $\mathrm{C}=3 \cdot 10^{-6} \mathrm{M}$. For our experiment, absorption and luminescence spectra of initially prepared QD solution are presented in Fig. 1; experimental exciton peak being at $523 \mathrm{~nm}$ in absorption and at $551 \mathrm{~nm}$ in the luminescence.

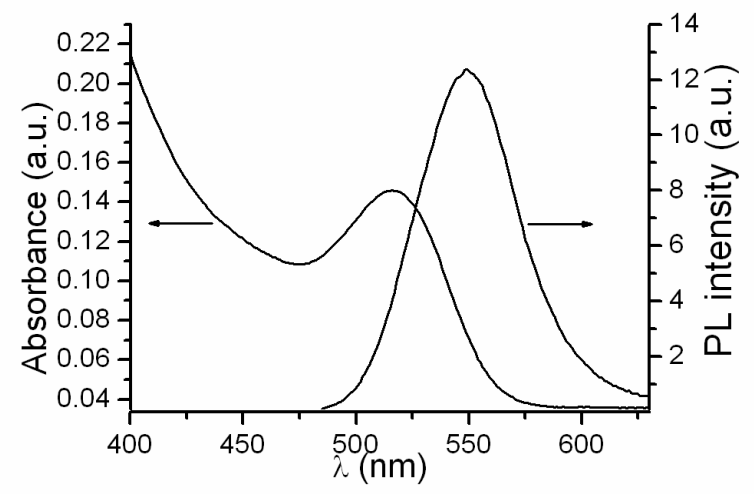

FIGURE 1. Optical density of $0.9 \mathrm{~cm}$ thick CdTe solution (Perkin-Elmer Lambda 35, left scale) and luminescence spectra excited at $480 \mathrm{~nm}$ (HoribaJobinIvon Fluorolog3, right scale).

Colloid solutions were illuminated using DRSh-250-3M mercury lamp. Irradiance spectrum of this lamp is plotted in Fig. 2 and contains a number of intense lines in the range from 275 to $600 \mathrm{~nm}$.

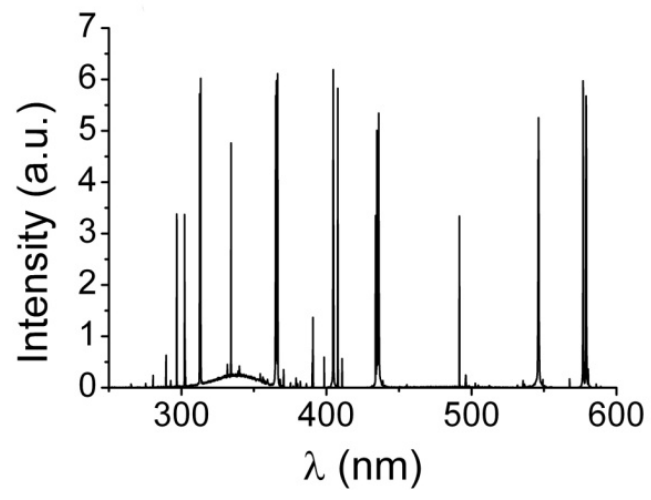

FIGURE 2. Spectra of DRSh -250-3M lamp used for CdTe QDs' solution illumination.

Power of radiation over the aperture of colloid solution was measured by thermopile light sensor and was found to be $\mathrm{P}=0.067 \mathrm{~W}$, which corresponds to the intensity of irradiating light $\mathrm{I}=0.4 \mathrm{~W} / \mathrm{cm}^{2}$. Irradiation of QDs' solutions was done in quartz cells. Maximum irradiation time was $150 \mathrm{~min}$, in order to reveal the complete dynamics of solutions' behavior, including the time period where aggregation becomes pronounced. 


\section{RESULTS AND DISCUSSION}

Action of radiation onto QDs' solutions can lead to destroying of either stabilizer molecules or QD's surfaces. Partial violation of stabilizer layer leads to spontaneous aggregation due to either Coulomb or van der Waals interaction. Degradation of QDs' surface leads to decrease of the size of initially dissolved ones. Both processes lead to the decrease of both absorption coefficient and luminescence intensity in the spectral ranges of first exciton transition, accompanied by changes of absorption spectra at shorter wavelengths.

To investigate the longer-time dynamics of CdTe QDs' solutions, we exposed them to mercury lamp irradiation with no filters used during 150 min. Temporal evolution of exciton absorption and luminescence magnitudes and exciton peak position in absorption and luminescence is depicted in Fig. 3.

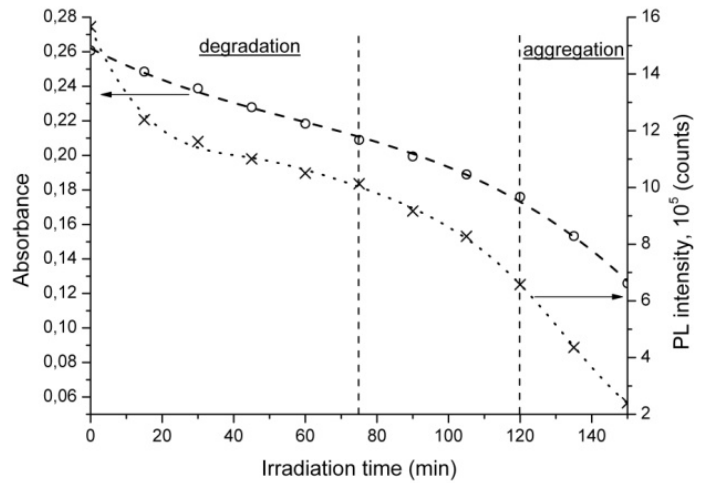

(a)

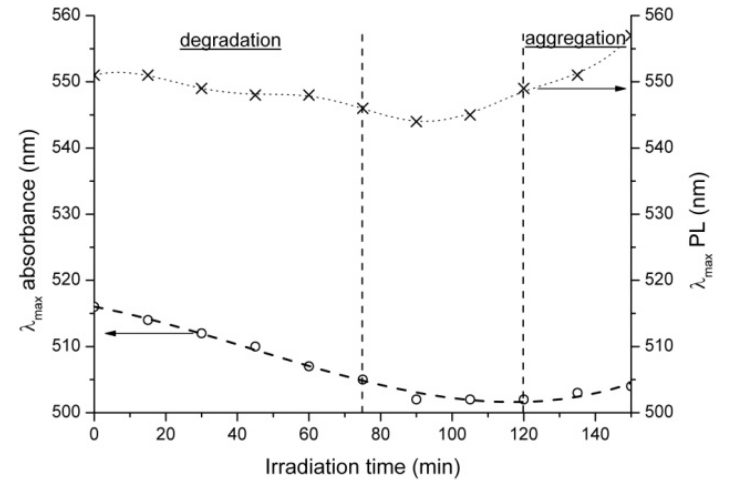

(b)

FIGURE 3. Dependences of magnitudes (a) and positions (b) of absorption and luminescence peaks on the UV+visible irradiation time.

After approximately 75 min of irradiation the decrease of magnitudes becomes faster, while positions of bands turn from the blue shift to the red shift after $100 \mathrm{~min}$ of irradiation. At the larger irradiation time, the formation of QDs' aggregates becomes well seen by the naked eye; these aggregates being sparce and probably of fractal structure. Shift of absorption of metal particles within aggregates, including fractal ones, is well known to be due to electrodynamic interaction between particles. This shift strongly depends on the environment of a particle, resulting in an inhomogeneous broadening of absorption band and to the redshift of its maximum accompanied by absorption magnitude decrease (see, e.g. [11]). Redshift of CdTe QDs luminescence maximum observed in our study after $100 \mathrm{~min}$ of irradiation evidently is due to the influence of electronynamic interaction within aggregates, too. One may expect appearance of additional mechanisms of radiativeless relaxation in aggregates, as well.

\section{CONCLUSION}

The height of the barrier preventing the spontaneous aggregation can be lowered under UV+visible irradiation. The case of semiconductor QDs is considerably more complicated than the case of metal nanoparticles, the latter being governed by the single mechanism, namely, photoelectron emission. Individual optical properties of metal nanoparticles, especially, their absorption spectra, remain untouched by the irradiation, and inhomogeneous broadening of absorption band is due to electrodynamical interaction of the particles within the fractal cluster. Strong influence of the stabilizer onto QDs' optical properties leads to fundamentally different character of lightinduced aggregation. Photochemical interactions lead to destruction of the stabilizer and of the QD's surface, and this leads to not only aggregation, but also to the alteration of the properties of individual particles. Thereby, laserinduced self-assembly of CdTe QDs is recommended to be performed in the time period from 80 to 100 min after the beginning of low intensity $\left(0.4 \mathrm{~W} / \mathrm{cm}^{2}\right) \mathrm{UV}$ irradiation in the conditions equivalent to those used in the present 
study. During this time interval, the height of aggregation-preventing barrier is already decreased while noticeable drop of QDs' content is still absent.

\section{ACKNOWLEDGMENTS}

This research is partially supported by grant of the Ministry of Education and Science of the Russian Federation for Siberian Federal University (3.6341.2017/BY) and by RFBR and Krasnoyarsk Krai according to the research projects No. 16-42-240410r_a, 17-42-240912r_a.

\section{REFERENCES}

1. L.J. Yu, K.J. Seon, P.J. Chul, N.Y. Sung, WIREs Nanomed Nanobiotechnol 8, 178-190 (2016).

2. B. Priem, C. Tian, J. Tang, Y. Zhao, W. JM. Mulder, Expert Opin. Drug Deliv. 12, 1881-1894 (2015).

3. S.M. Ng, M. Koneswaran, R. Narayanaswamy, RSCAdv. 6, 21624 -21661 (2016).

4. T.H. Kim, K.S. Cho, E.K. Lee, S.J. Lee, J. Chae, J.W. Kim, D.H. Kim, J.Y. Kwon, G. Amaratunga, S.Y. Lee, B.Y. Choi, Y. Kuk, J.M. Kim, K. Kim, Nat. Photon. 5, 176-182 (2011).

5. Y. Yang, Y. Zheng, W. Cao, A. Titov, J. Hyvonen, J.R. Manders, J. Xue, P.H. Holloway, L. Qian, Nat. Photon. 9, 259-266 (2015).

6. D. Zhao, C.F. Yang, Renew Sust Energ Rev 54, 1048-1059 (2016).

7. V.V. Slabko, A.S. Tsipotan, A.S. Aleksandrovsky, Photonics and nanostructures-fundamentals and applications 10, 636-643 (2012).

8. A.S. Tsipotan, M.A. Gerasimova, V.V. Slabko and A.S. Aleksandrovsky, Optics Express 24, 11145-11150 (2016).

9. S.V. Karpov, A.K. Popov and V.V. Slabko, JETP Lett. 66, 106-110 (1997).

10. S.V. Karpov, V.V. Slabko and G.A. Chiganova, Colloid Journal 64, 425-441 (2002).

11. S.V. Karpov, A.L. Bas'ko, A.K. Popov, V.V. Slabko, Colloid Journal 62, 699-713 (2000). 